\title{
Regularized inversion of aerosol hygroscopic growth factor probability density function: Application to humidity-controlled fast integrated mobility spectrometer measurements
}

\author{
Jiaoshi Zhang ${ }^{1}$, Yang Wang ${ }^{1,3}$, Steven Spielman ${ }^{2}$, Susanne Hering ${ }^{2}$, and Jian Wang ${ }^{1}$
}

$5 \quad{ }^{1}$ Center for Aerosol Science and Engineering, Washington University in St. Louis, St. Louis, Missouri, USA

${ }^{2}$ Aerosol Dynamics Inc, Berkeley, California, USA

${ }^{3}$ Department of Civil, Architectural and Environmental Engineering, Missouri University of Science and Technology, Rolla, Missouri, USA

Correspondence to: Jian Wang (jian@wustl.edu)

10 Abstract. Aerosol hygroscopic growth plays an important role in atmospheric particle chemistry and the effects of aerosol on radiation and hence climate. The hygroscopic growth is often characterized by a growth factor probability density function (GF-PDF), where the growth factor is defined as the ratio of the particle size at a specified relative humidity to its dry size. Parametric, least-square methods are the most widely used algorithms for inverting the GF-PDF from measurements of humidified tandem differential mobility analyzers (HTDMA) and have been recently applied to the GF-

15 PDF inversion from measurements of the humidity-controlled fast integrated mobility spectrometer (HFIMS). However, these least square methods suffer from noise amplification due to the lack of regularization in solving the ill-posed problem, resulting in significant fluctuations in the retrieved GF-PDF and even occasional failures of convergence. In this study, we introduce nonparametric, regularized methods to invert aerosol GF-PDF and apply them to HFIMS measurements. Based on the HFIMS kernel function, the forward convolution is transformed into a matrix-based form, which facilitates the application of the nonparametric inversion methods with regularizations, including Tikhonov regularization and Twomey's iterative regularization. Inversions of the GF-PDF using the nonparameteric methods with regularization are demonstrated using HFIMS measurements simulated from representative GF-PDFs of ambient aerosols. The characteristics of reconstructed GF-PDFs resulting from different inversion methods, including previously developed least-square methods, are quantitively compared. The result shows that Twomey's method generally outperforms other inversion methods. The capabilities of Twomey's method in reconstructing the pre-defined GF-PDFs and recovering the mode parameters are validated.

\section{Introduction}

The hygroscopic growth of aerosol particles influences heterogeneous reactions, light extinction, and visibility, whereby aerosol water is most relevant for the direct radiative forcing of Earth's climate (Tang and Munkelwitz, 1994; Pilinis et al., 1995; Swietlicki et al., 2008). The ability of aerosols to absorb water depends mainly on their compositions, hence the 
hygroscopic properties reflect the variability of the key chemical components (Gysel et al., 2007; Zheng et al., 2020). Therefore, the variation of aerosol hygroscopic growth can be used to infer the potential chemical composition, especially for small aerosols that are beyond the size range of the aerosol mass spectrometer. Aerosol hygroscopic growth under atmospheric relative humidity ( $\mathrm{RH})$ is commonly measured by a humidified tandem differential mobility analyzer system (HTDMA) (Liu et al., 1978; Rader and McMurry, 1986; Swietlicki et al., 2008). In an HTDMA system, monodisperse particles classified by the $1^{\text {st }}$ DMA are exposed to an elevated RH in a humidity conditioner, and the size distribution of humidified particles is then measured by a $2^{\text {nd }}$ DMA and a particle detector using scanning mobility technique. The particle hygroscopic growth is then derived from the size distribution of the humidified particles. Recently, a humidity-controlled fast integrated mobility spectrometer (HFIMS) was developed. The HFIMS replaces the $2^{\text {nd }}$ DMA and particle detector

40 within the HTDMA system with a water-based fast integrated mobility spectrometer (WFIMS), which captures the size distribution of humidified particles instantly (Pinterich et al., 2017a). As a result, the HFIMS drastically accelerates aerosol hygroscopic growth measurements (Pinterich et al., 2017b; Wang et al., 2019; Zhang et al., 2021), making it feasible to characterize ambient aerosol hygroscopic growth at a wide range of sizes and RH levels under $\sim 25$ min.

The HTDMA measurement, i.e., the mobility-concentration distribution of humidified particles, is a convolution of the

45 aerosol hygroscopic growth factor probability density function (GF-PDF) and the transfer functions of both DMAs. Similarly, the HFIMS measurement represents a convolution of the aerosol GF-PDF together with the transfer functions of the DMA and the WFIMS (Wang et al., 2019). Two inversion algorithms, TDMAfit (Stolzenburg and McMurry (1988)) and TDMAinv (Gysel et al. (2009)) were developed and widely used to retrieve the GF-PDF from HTDMA measurements. In both algorithms, the GF-PDF is represented with a specific functional form, and the function parameters were derived by 50 least-squares fitting. For example, the TDMAfit algorithm assumes the GF-PDF as a superposition of multiple Gaussian distribution functions (Stolzenburg and McMurry, 1988) or a summation of multiple lognormal (ML) distribution functions (Stolzenburg and McMurry, 2008). Likewise, TDMAinv describes the GF-PDF as a piecewise linear (PL) function at predefined growth factor values (Gysel et al., 2009). The function parameters are derived using least-squares fitting that minimizes the residual between the measured and reconstructed size distributions of humidified particles. Similar methods

55 have been applied to invert GF-PDFs from HFIMS measurements by Wang et al. (2019).

Inversion of the GF-PDF from the HTDMA or HFIMS measurements is an ill-posed problem (Gysel et al., 2009). Leastsquares methods such as TDMAfit and TDMAinv provide simple and effective ways to solve this ill-posed problem by representing the GF-PDF in a specific functional form (Kandlikar and Ramachandran, 1999). However, the GF-PDF inverted by the TDMAfit algorithm often relies on the initial guess of the parameters, resulting in occasional failures of convergence

60 (Gysel et al., 2009). For example, it was reported that the TDMAfit algorithm may not be robust in cases of closely multiple overlapped modes and the successful convergence depends on the initial guess (Swietlicki et al., 2008). Moreover, it is wellknown that the unregularized least-squares method amplifies the measurement noise (Kandlikar and Ramachandran, 1999; Sipkens et al., 2020), resulting in significant fluctuations in the retrieved GF-PDF. It has been shown that the derived GFPDF using the TDMAinv algorithm may oscillate strongly when a higher bin resolution is chosen, while a too low resolution 

interpretation of the aerosol mixing state (Wang et al., 2019). The approach to overcoming noise amplification is to regularize the problem by including additional information, such as smoothness (Kandlikar and Ramachandran, 1999). Tikhonov regularization is among the most common regularization methods and has been applied to inversions of the aerosol size distribution (Talukdar and Swihart, 2003) and mass-mobility distribution (Sipkens et al., 2020). Recently, a software package was developed to invert HTMDA data using Tikhonov regularization (Petters, 2021). Twomey's algorithm (Twomey, 1975), one of the most common iterative regularization methods, has been widely used to invert aerosol size distributions (Collins et al., 2002; Olfert et al., 2008; Wang et al., 2018) and two-dimensional mass-mobility distributions (Rawat et al., 2016; Sipkens et al., 2020). However, to our best knowledge, the Twomey's algorithm has not been applied to invert GF-PDF from HTDMA or HFIMS measurements.

75 In this study, we present nonparametric, regularized inversions of the GF-PDF from HFIMS measurements. These inversion methods can be adapted to HTMDA measurements straightforwardly. The forward model (i.e., the convolution of the GFPDF, the transfer function of DMA, and the transfer function of WFIMS) is derived analytically and cast into a matrix form such that nonparametric inversion methods can be conveniently applied. The nonparametric inversions are demonstrated by retrieving GF-PDF from HFIMS measurements of ambient aerosols. The dependence of retrieved GF-PDF on GF bin

80 resolutions is investigated, and an optimal GF bin resolution is identified. Synthetic data are generated using representative GF-PDFs of ambient aerosols and are applied to evaluate different inversion methods, including (1) parametric, least-squares fittings, (2) nonparametric, unregularized least-squares, (3) Twomey's algorithm, and (4) Tikhonov regularization. The performances of the different inversion methods including reconstruction accuracy, GF-PDF fidelity, smoothness, and computation time are presented and discussed.

\section{Methods}

This section presents the GF-PDF inversion routine from the HFIMS measurement, which includes the mathematical derivation of the matrix-based inverse problem, the description of different inversion algorithms, and the generation of synthetic data for evaluating the inversion algorithms.

\subsection{A matrix form for the forward model}

90 The integrated response of HFIMS is determined by the aerosol size distribution, the DMA transfer function, the GF-PDF, and the WFIMS transfer function Wang et al. (2019). The number concentration of particles with diameters between $D_{p 1}$ and $D_{p 1}+\mathrm{d} D_{p 1}$ downstream of the DMA inside the HFIMS is given by:

$$
\mathrm{d} N_{\mathrm{DMA}}=\frac{Q_{\mathrm{a}, \mathrm{DMA}}}{Q_{\mathrm{s}, \mathrm{DMA}}} \eta_{\mathrm{chg}}\left(D_{\mathrm{p} 1}\right) \eta_{\mathrm{p}, \mathrm{DMA}}\left(D_{\mathrm{p} 1}\right) \Omega\left(V_{\mathrm{DMA}}, \tilde{Z}_{\mathrm{p} 1}\right) \mathrm{d} N
$$


where $Q_{\mathrm{a}, \mathrm{DMA}}$ and $Q_{\mathrm{s}, \mathrm{DMA}}$ are the DMA aerosol and sample (i.e., monodispersed) flow rates, respectively, $\eta_{\mathrm{chg}}\left(D_{\mathrm{p} 1}\right)$ is the aerosol charging efficiency, $\eta_{\mathrm{p}, \mathrm{DMA}}\left(D_{\mathrm{p} 1}\right)$ is the particle penetration efficiency through the DMA, and $\Omega\left(V_{\mathrm{DMA}}, \tilde{Z}_{\mathrm{p} 1}\right)$ is the transfer function of the DMA operated with the classifying voltage of $V_{\mathrm{DMA}}, \tilde{Z}_{\mathrm{p} 1}$ is the particle mobility $\left(Z_{\mathrm{p} 1}\right)$ normalized by the DMA centroid mobility corresponding to $V_{\mathrm{DMA}} . \mathrm{d} N=n\left(D_{\mathrm{p} 1}\right) \mathrm{d} D_{\mathrm{p} 1}$ represents the number concentration of particles with diameters between $D_{\mathrm{p} 1}$ and $D_{\mathrm{p} 1}+\mathrm{d} D_{\mathrm{p} 1}$. The number concentration of particles with diameters between $D_{\mathrm{p} 2}$ and $D_{\mathrm{p} 2}+\mathrm{d} D_{\mathrm{p} 2}$ at the outlet of the conditioner is

$$
\mathrm{d} N_{\text {cond }}=\mathrm{d} D_{\mathrm{p} 2} \int_{D_{\mathrm{p} 1}=0}^{D_{\mathrm{p}}=\infty} \eta_{\mathrm{p}, \text { cond }}\left(D_{\mathrm{p} 2}\right) c_{\text {cond }}\left(D_{\mathrm{p} 2}, D_{\mathrm{p} 1}\right) \mathrm{d} N_{\text {DMA }}
$$

where the integration considers all possible values of $D_{\mathrm{p} 1} \cdot \eta_{\mathrm{p}, \text { cond }}\left(D_{\mathrm{p} 2}\right)$ is the penetration efficiency of the conditioned particles, assuming the particle growth from $D_{\mathrm{p} 1}$ to $D_{\mathrm{p} 2}$ is instantaneous. $c_{\text {cond }}\left(D_{\mathrm{p} 2}, D_{\mathrm{p} 1}\right)$ is the growth factor probability density function (GF-PDF) for particles with a dry diameter of $D_{\mathrm{p} 1}$ growing to a diameter of $D_{\mathrm{p} 2}$ during the humidity conditioning process. The GF-PDF satisfies $\int_{D_{\mathrm{p} 2}=0}^{D_{\mathrm{p}}=\infty} c_{\text {cond }}\left(D_{\mathrm{p} 2}, D_{\mathrm{p} 1}\right) \mathrm{d} D_{\mathrm{p} 2}=1$.

105 The WFIMS response to particles with diameters between $D_{\mathrm{p} 2}$ and $D_{\mathrm{p} 2}+\mathrm{d} D_{\mathrm{p} 2}$ in the $i^{t h} D_{\mathrm{p}}^{*}$ bin during any time interval $(t)$ is calculated by

$$
\mathrm{d} R_{i}=\frac{Q_{\mathrm{a}, \mathrm{WFIMS}} N_{F}}{\dot{N}_{F}} \eta_{\mathrm{p}, \mathrm{WFIMS}}\left(D_{\mathrm{p} 2}\right) \eta_{\mathrm{det}}\left(D_{\mathrm{p} 2}\right) \Omega_{\mathrm{WFIMS}, i}\left(Z_{\mathrm{p} 2}\right) \mathrm{d} N_{\text {cond }}
$$

$Q_{\mathrm{a}, \mathrm{WFIMS}}$ is the inlet flow rate through the WFIMS, $N_{F}$ is the number of frames being used to count $\mathrm{d} R_{i}, \dot{N}_{F}$ is the frame rate. $N_{F} / \dot{N}_{F}$ represents the time interval $(t)$ of counting, $\eta_{\mathrm{p}, \text { WFIMS }}$ is the penetration efficiency of particles going through the WFIMS separator, $\Omega_{\mathrm{WFIMS}, i}\left(Z_{\mathrm{p} 2}\right)$ is the transfer function of the $i^{\text {th }}$ bin of the instrument response diameter $\left(D_{\mathrm{p}}^{*}\right)$ of the WFIMS. Note that the detection efficiency for particles above $8 \mathrm{~nm}$ has been shown to be 1 (i.e., $\eta_{\mathrm{det}}\left(D_{\mathrm{p} 2}\right)=1$, Pinterich et al., 2017a).

The theoretical response of the $i^{\text {th }} D_{\mathrm{p}}^{*}$ bin of the HFIMS, $R_{i}$, can be derived by combining the above equations as detailed in

115 Wang et al. (2019):

$$
R_{i}=E \iint \frac{1}{D_{\mathrm{p} 1}} c_{\text {cond }}\left(g, D_{\mathrm{p} 1}\right) \Omega\left(V_{\mathrm{DMA}}, \tilde{Z}_{\mathrm{p} 1}\right) \Omega_{\mathrm{WFIMS}, i}\left(Z_{\mathrm{p} 2}\right) \mathrm{d} D_{\mathrm{p} 2} \mathrm{~d} D_{\mathrm{p} 1}
$$

Where $E=\left.R_{\text {tot }} \frac{b}{b_{\text {view }}} \frac{Q_{s h, \mathrm{DMA}}}{Q_{a, \mathrm{DMA}}} \frac{d \tilde{z}_{\mathrm{p} 1}}{d D_{\mathrm{p} 1}}\right|_{D_{\mathrm{p} 1}^{*}} . R_{\text {tot }}$ is the total counts of particles detected within the WFIMS viewing window, i.e., $R_{\mathrm{tot}}=\sum_{i} R_{\mathrm{i}}$, where $R_{\mathrm{i}}$ is the response of the $i^{\text {th }} D_{\mathrm{p}}^{*}$ bin of the WFIMS. $b_{\text {view }}$ and $b$ are the length of the viewing area of the CCD-captured image and the length of the WFIMS mobility separator. In Eq. (4), the GF-PDF is written as a function of growth factor $g$ (i.e., $\left.D_{\mathrm{p} 2} / D_{\mathrm{p} 1}\right)$, and it satisfies $c_{\text {cond, } \mathrm{n}}\left(g, D_{\mathrm{p} 1}\right) d g=c_{\text {cond }}\left(D_{\mathrm{p} 2}, D_{\mathrm{p} 1}\right) d D_{\mathrm{p} 2}$. Given the narrow particle size range classified by the DMA, we assume the GF-PDF is the same for all particles classified by the DMA at a given voltage, 
i.e., $c_{\text {cond }}\left(g, D_{p 1}\right)$ is independent of $D_{\mathrm{p} 1}$ for the integration in Eq. (4). Rewriting the GF-PDF as $c_{\text {cond }}(g)$ and replacing $D_{\mathrm{p} 2}$ with $g D_{\mathrm{p} 1}$ in Eq. (4) gives:

$$
R_{i}=E \int_{0}^{+\infty} d g c_{\text {cond }}(g) \int_{0}^{+\infty} d D_{\mathrm{p} 1} \Omega\left[V_{\mathrm{DMA}}, \tilde{Z}_{\mathrm{p} 1}\left(D_{\mathrm{p} 1}\right)\right] \Omega_{\mathrm{WFIMS}, i}\left[Z_{\mathrm{p}}\left(g D_{\mathrm{p} 1}\right)\right]
$$

125 The integration can be written as a sum over $J$ GF bins, with the assumption that $c_{\text {cond }}(g)$ is a constant value within each GF bin:

$$
R_{i, \text { theo }}=E \sum_{j=1}^{J} c_{\text {cond }}\left(g_{j}\right) \int_{g_{j-\frac{1}{2}}}^{g} d g \int_{0}^{+\infty} d D_{\mathrm{p} 1} \Omega\left[V_{\mathrm{DMA}}, \tilde{Z}_{\mathrm{p}}\left(D_{\mathrm{p} 1}\right)\right] \Omega_{\mathrm{WFIMS}, i}\left[Z_{\mathrm{p}}\left(g D_{\mathrm{p} 1}\right)\right]
$$

where $g_{j-1 / 2}$ and $g_{j+1 / 2}(j=1,2,3, \ldots, J)$ are the lower and upper bounds of the $j$ th GF bin. Eq. (6) can be further arranged into a matrix form as

$$
\mathbf{R}=\mathbf{M} \times \mathbf{c}
$$

where the HFIMS response $\mathbf{R}$ is an $I \times 1$ array composed of $R_{i}(i=1,2,3, \ldots, I)$. $I$ is the selected size bins of the WFIMS that covers the size range of $\left(0.8 D_{\mathrm{p} 1}^{*}, 2.0 D_{\mathrm{p} 1}^{*}\right)$ according to the settings of the DMA centroid diameter $D_{\mathrm{p} 1}^{*}$. The unknown GF-PDF $\mathbf{c}$, an $J \times 1$ array composed of $c_{j}(j=1,2,3, \ldots, J)$, can be found by solving the Fredholm integral equation (7).

The element of the HFIMS kernel matrix, $\mathbf{M}$, is calculated by

$$
M_{i j}=E_{i} \int_{g_{j-\frac{1}{2}}}^{g}+\frac{1}{2} d g \int_{0}^{+\infty} d D_{\mathrm{p} 1} \Omega\left[V_{\mathrm{DMA}}, \tilde{Z}_{\mathrm{p}}\left(D_{\mathrm{p} 1}\right)\right] \Omega_{\mathrm{WFIMS}, i}\left[Z_{\mathrm{p}}\left(g D_{\mathrm{p} 1}\right)\right]
$$

The HFIMS kernel describes the probability of particles with GF between $g_{j-1 / 2}$ and $g_{j+1 / 2}$ that is measured between the channel limits between $Z_{\mathrm{p}, i-1 / 2}^{*}$ and $Z_{\mathrm{p}, i-1 / 2}^{*}$. As described above, the inversion of the GF-PDF (c) becomes an ill-posed problem due to overlapping of the HFIMS kernel function, like that of the aerosol size spectrometers (Kandlikar and Ramachandran, 1999; Collins et al., 2002; Talukdar and Swihart, 2003). It is worth noting that the derivation of the HFIMS kernel function can be easily applied to HTDMA measurement by replacing the WFIMS transfer function $\Omega_{\mathrm{WFIMS}, i}\left[Z_{p}\left(g D_{\mathrm{p} 1}\right)\right]$ with the transfer function of the $2^{\text {nd }}$ DMA $\Omega\left[V_{i}, Z_{p}\left(g D_{\mathrm{p} 1}\right)\right]$ in Eq. (8), where $V_{i}$ is the classifying voltage of the $2^{\text {nd }}$ DMA.

\subsection{Inversion methods}

A number of techniques have been developed to solve the Fredholm integration (Kandlikar and Ramachandran, 1999). With

145 Eqs. (7) and (8), nonparametric algorithms can be straightforwardly applied to invert GF-PDF, hence no prior knowledge of the functional form of GF-PDF is needed.

\section{Unregularized least-squares}

The simplest route is the ordinary least-squares (LSQ) which seeks to minimize the square of the residual:

$$
\mathbf{c}^{L S Q}=\arg \min _{\mathbf{c}}\left\{\|\mathbf{M c}-\mathbf{R}\|_{2}^{2}\right\}
$$


150 where $\|\cdot\|_{2}$ denotes the Euclidean norm. Here, the least-squares solution is solved by using the lsqnonneg function from MATLAB.

\section{Tikhonov regularization}

Tikhonov regularization is a common regularization method that overcomes noise amplification, and it has been used to invert aerosol size distribution and 2-D aerosol mass-mobility distributions (Talukdar and Swihart, 2003; Sipkens et al.,

155 2020). In Tikhonov regularization, an additional regularization term is included in the least-squares approach:

$$
\mathbf{c}^{\text {Tik }}=\arg \min _{\mathbf{c}}\left\{\|\mathbf{M c}-\mathbf{R}\|_{2}^{2}+\lambda^{2}\|\mathbf{L c}\|_{2}^{2}\right\}
$$

where $\lambda^{2}\|\mathbf{L} \mathbf{c}\|_{2}^{2}$ represents the regularization term designed to minimize the derivative of a specific order and $\lambda$ is the regularization parameter that controls the degree of regularization. The penalization matrix $\mathbf{L}$ is often set as the identity matrix I, the bidiagonal $(-1,1)$ matrix, and the upper tridiagonal $(1,-2,1)$ matrix for the $0^{\text {th }}, 1^{\text {st }}$, and $2^{\text {nd }}$ order regularization,

160 respectively (Hansen and O’Leary, 1993; Hansen, 1994). The parametric L-curve of $\left\|\mathbf{M} \mathbf{c}_{\lambda}-\mathbf{R}\right\|_{2}$ vs $\left\|\mathbf{L} \mathbf{c}_{\lambda}\right\|_{2}$ is plotted and the corner of the L-curve is identified as the optimal regularization parameter $\lambda$ which corresponds to a good balance between minimization of the residual and reduction of the noise level (Hansen, 1992; Hansen and O’Leary, 1993).

\section{Twomey's method}

Twomey's method is commonly used to find solutions for ill-posed problems and has been proved to be effective in

165 inversions of the aerosol size distribution (Collins et al., 2002; Olfert et al., 2008) and aerosol mass-mobility distribution (Rawat et al., 2016; Sipkens et al., 2020). It is a non-linear optimization method and provides iterative regularizations. An initial guess solution is iteratively multiplied by small multiples of the HFIMS kernel function which are proportional to the ratio of the measured to calculated measurements as follows:

$$
c_{j}^{k+1}=\left[1+\left(\frac{R_{i}}{\mathbf{m}_{i} \mathbf{c}^{k}}-1\right) M_{i j}\right] \cdot c_{j}^{k}
$$

170 where $\mathbf{m}_{i}$ is the $i$ th row of the HFIMS kernel function $\mathbf{M}$, and $R_{i} / \mathbf{m}_{i} \mathbf{c}^{k}$ denotes the relative divergence between actual and reconstructed HFIMS measurements. The positively constrained, least-squares solution is set as the initial guess (Olfert et al., 2008). Then, the initial guess is smoothed using a three-term moving average (Markowski, 1987) and input into the iterative Twomey's routine which is then repeated until a Chi-squared criterion is satisfied.

\section{Parametric LSQ fittings}

175 The parametric fitting methods assume a prior known distribution of the GF-PDF and calculate the forward model problem (Eq. 4) to reconstruct the HFIMS measurements. A nonlinear least-squares fitting with boundary constraints is performed to search for the least-squares solution within the bounds. The ML and PL fitting routines for the GF-PDF inversion from HFIMS measurements have been developed by Wang et al. (2019). The influence of counting statistics and GF-PDF parameters (i.e., the number of modes of ML GF-PDF and the number of sections of PL GF-PDF) has been statistically 
180 studied. In this work, the GF-PDF inverted using ML and PL fitting routines with the optimized parameters are compared with those retrieved using nonparametric inversion methods described above.

\subsection{Generation of synthetic data to evaluate inversion algorithms}

HFIMS measurements are synthesized to evaluate the performance of different inversion methods. The synthetic data are based on three representative GF-PDF that consist of one, two, and three lognormal modes, respectively. The mode parameters of the pre-defined GF-PDFs are listed in Table 1, similar to those listed in Wang et al. (2019). The parameters of $f, G, \sigma$ are the fractional weight, mean diameter growth factor, and geometric standard deviation of each mode. The theoretical HFIMS response (i.e., $R_{i}$ ) is first derived using the Eq. (4) based on each of the three GF-PDFs. The actual FIMS measurement follows the following Poisson distribution:

$$
P(x)=\frac{R_{i}^{x}}{x !} \exp \left(-R_{i}\right)
$$

190 where $R_{i}$ is the derived theoretical response of the $i^{\text {th }} D_{\mathrm{p}}^{*}$ bin, and $P(x)$ is the probability that $x$ number of particles are detected by HFIMS in the $i^{\text {th }} D_{\mathrm{p}}^{*}$ bin (i.e., actual measurements). Five hundred sets of HFIMS measurements $\left(R_{i}\right)$ are generated using Monte Carlo methods based on the Poisson distribution for each $D_{\mathrm{p}}^{*}$ bin. Note that the total particle counts detected during a typical HFIMS ambient measurement period (i.e., at least 20 seconds, Zhang et al., 2021) $R_{\text {tot }}$ are set to 100 to maintain a constant counting statistic. These synthetic HFIMS measurements are used to evaluate the inversion methods described above.

Table 1. Mode parameters of representative GF-PDFs for generating synthetic HFIMS measurements.

\begin{tabular}{cccccccccc}
\hline $\begin{array}{c}\text { Predefined } \\
\text { GF-PDF }\end{array}$ & $f$ & $G$ & $\sigma$ & $f$ & $G$ & $\sigma$ & $f$ & $G$ & $\sigma$ \\
\hline 1 & 1.0 & 1.40 & 1.15 & & NA & & & NA \\
2 & 0.45 & 1.10 & 1.05 & 0.55 & 1.30 & 1.05 & & NA & \\
3 & 0.39 & 1.05 & 1.10 & 0.32 & 1.40 & 1.05 & 0.29 & 1.70 & 1.10 \\
\hline
\end{tabular}

\section{Results and discussion}

\subsection{Optimal numbers of Growth factor bins and HFIMS size bins $\left(D_{\mathrm{p}}^{*}\right)$}

The numbers of GF bins $(J)$ and $D_{\mathrm{p}}^{*}$ bins $(I)$ determine the dimensions of HFIMS kernel function, which affects the inversion of GF-PDF. The optimal number of $D_{\mathrm{p}}^{*}$ bin is a trade-off between sizing resolution and counting statistics. Wang et al. (2019) examined the influence of WFIMS $D_{\mathrm{p}}^{*}$ bin number $(I)$ on the inverted GF-PDF and found that an optimal range of 23-32 for a total particle counts of 100. For representative remote continental and urban aerosols, the number of particles measured by the HFIMS often exceed 100 in 20 seconds (Pinterich et al., 2017b; Zhang et al., 2021). The dynamic range of WFIMS is roughly a factor of 10 in mobility, corresponding to a factor of $\sim 3$ in the size range (Zhang et al., 2021). In this 
study, 30 size bins (i.e., $I=30$ ) that are evenly spaced on a logarithmic scale over the WFIMS size range are used in the inversions.

The influence of growth factor bin number $(J)$ on the inverted GF-PDF is examined using the synthetic HFIMS measurements described above. The GF-PDF was inverted from each set of the simulated HFIMS measurements using different GF bin numbers ranging from 10 to 50 (i.e., corresponding to a GF resolution range of 0.024 - 0.12). To facilitate the comparison of GF-PDFs inverted with different GF bin numbers, we interpolate the inverted GF-PDFs to 120 fixed growth factors that are evenly distributed from 0.8 to 2.0. The average error of the inverted GF-PDF $\gamma$ is defined as:

$$
\gamma^{2}=\frac{1}{N} \sum_{i=1}^{N}\left(c_{i, i n v}-c_{i, s i m}\right)^{2}
$$

where $c_{i, i n v}$ and $c_{i, s i m}$ are the interpolated GF-PDF and pre-defined GF-PDF (i.e., true values) at the 120 fixed growth factors, respectively. $N$ is the number of points of fixed growth factors (i.e., 120). The smoothness of the inverted GF-PDF is evaluated using the absolute second-order derivative:

$$
\xi=\sum_{i=2}^{N-1}\left|2 c_{i n v}\left(g_{i}\right)-c_{i n v}\left(g_{i+1}\right)-c_{i n v}\left(g_{i-1}\right)\right|
$$

To evaluate how well the inverted GF-PDF reproduces the HFIMS measurement, we define the residual of the reconstructed FIMS measurement (i.e., reconstruction error) as:

$$
\chi^{2}=\sum_{i=1}^{L}\left(\tilde{R}_{i, i n v}-\tilde{R}_{i}\right)^{2}
$$

220 where $\tilde{R}_{i, i n v}$ is the normalized FIMS measurement that is reconstructed using Eq. (7) (i.e., forward calculation). $\tilde{R}_{i}$ is the normalized synthetic HFIMS measurement (i.e., true values).

Figure 1 shows the smoothness of the GF-PDF inverted using Twomey methods $(\xi)$ versus the residual of reconstructed HFIMS measurement $\left(\chi^{2}\right)$ for different GF bin numbers $(J)$. The variation of $\xi$ with $\chi^{2}$ exhibits an L-shaped curve for all three representative PF-PDF. The initial increase of $J$ from 10 to 20 substantially improves the agreement between the reconstructed and simulated HFIMS measurements, as indicated by a much reduced $\chi^{2}$ value. At the same time, $\xi$ remains relatively small, indicating a high smoothness of the inverted GF-PDF. In contrast, an increase of $J$ above 20 leads to a minor reduction of $\chi^{2}$ value but a drastic increase of $\xi$, suggesting strong noise in inverted GF-PDF. The optimal solution lies near the corner of the "L-curve" (Hansen and O'Leary, 1993) that strikes a balance between smoothness and fidelity to the HFIMS measurements. For all three pre-defined GF-PDFs, the corner of the L-curve corresponds to a $J$ value of 20. GF-PDF inverted with 20 growth factor bins generally shows the smallest error $\left(\gamma^{2}\right)$, indicating best agreements between the inverted and the true GF-PDFs. The same type of L-curves for GF-PDFs inverted using unregularized LSQ and Tikhonov regularization are shown in the supplementary information (SI), and they also reveal a corner that corresponds to a $J$ value of 20. These results suggest an optimal $J$ value of 20 for a range of representative GF-PDFs and different inversion methods. 

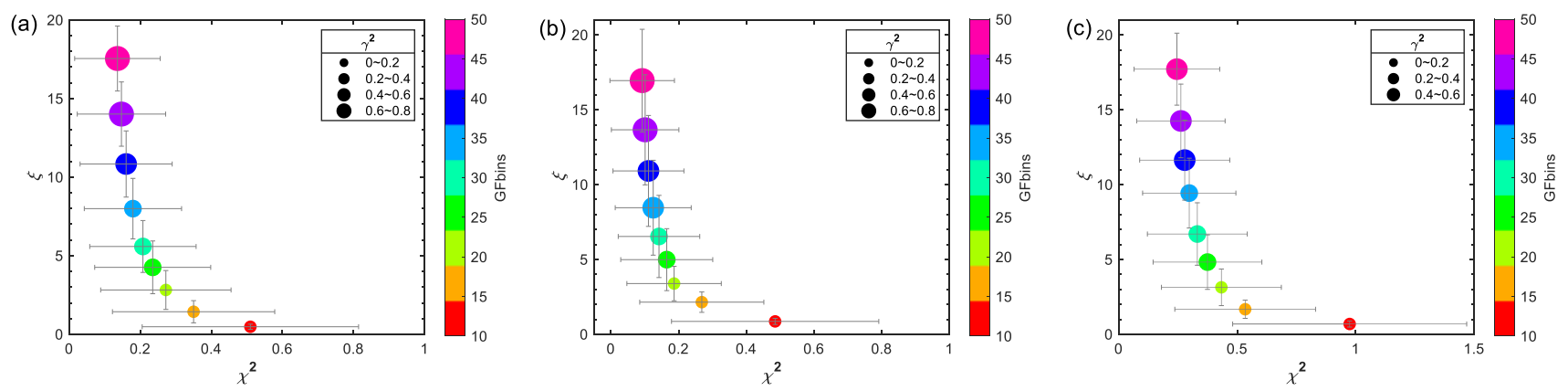

235 Figure 1. "L-curve" showing the dependence of reconstruction residual, $\chi^{2}$, and the smoothness, $\xi$, on the number of GF bins of predefined GF-PDFs with (a) one mode, (b) two modes, and (c) three modes, respectively. The symbol size represents the error in inverted GF-PDF, $\gamma^{2}$. Whiskers represent standard deviation. The inversion is conducted using Twomey's method.

\subsection{Comparisons of different inversion methods}

In this section, we systematically compare the performance of different inversion methods described in Section 2.2. For all nonparametric methods, the inversions were carried out using the optimal numbers of GF bins $(J)$ and $D_{\mathrm{p}}^{*}$ bins $(I), 20$ and 30 , respectively. Figure 2 shows the residual of reconstructed HFIMS measurements $\left(\chi^{2}\right)$, the smoothness $(\xi)$, the error of inverted GF-PDF residual $\left(\gamma^{2}\right)$, and the computing time for different inversion methods. The results are the averages for the 500 sets of synthetic HFIMS data are shown for each of three pre-defined GF-PDFs. Compared with parametric counterparts (i.e., ML and PL least-squares fitting), the nonparametric methods generally retrieve more accurate GF-PDFs. Note that the

245 ML least-squares fitting fails to converge to a valid solution occasionally, resulting in the abnormally large error in the inverted GF-PDFs, particularly for the pre-defined GF-PDFs with two and three modes. It may be due to the assumed spectral shape of GF-PDFs or the finite range of the boundary constraints that lead to a failure of searching for a leastsquares solution in the presence of random noise. Among all nonparametric inversion methods, the unregularized LSQ provides the solution with the lowest reconstruction residual but largest noise and error in the inverted GF-PDFs, consistent with the noise amplification in unregularized methods. In comparison, regularized inversion methods generally produce smoother solutions at the expense of increased reconstruction residuals. Among different Tikhonov regularization methods, higher-order regularizations (i.e., $1^{\text {st }}$ and $2^{\text {nd }}$ ) tend to produce smoother solutions, although the errors in inverted GF-PDF are very similar statistically. The $\xi$ value of the GF-PDF inverted using $1^{\text {st }}$ and $2^{\text {nd }}$ order Tikhonov regularizations increase with the mode number of GF-PDF, consistent with the increasingly more complex spectral shape of GF-PDF. Overall, Twomey's method outperforms the other regularized inversion methods regardless of the shapes of the pre-defined GF-PDFs. On average, the GF-PDF inverted using Twomey's method has the smallest error $\left(\gamma^{2}\right)$ and lowest $\xi$ value, indicative of the outstanding de-noising performance.

Fig. 2(d) shows that once the matrix is generated, the implementation of the nonparametric methods requires a much shorter computing time than the parametric fitting methods. Here, the computing time is recorded on a desktop with Intel's 8th 
generation processor Core i7-8700. On average, a single-time implement of any nonparametric method requires only $\sim 1$ s for all three pre-defined GF-PDFs. In contrast, both ML and PL least-squares fitting routines require more than one order of magnitude longer time.
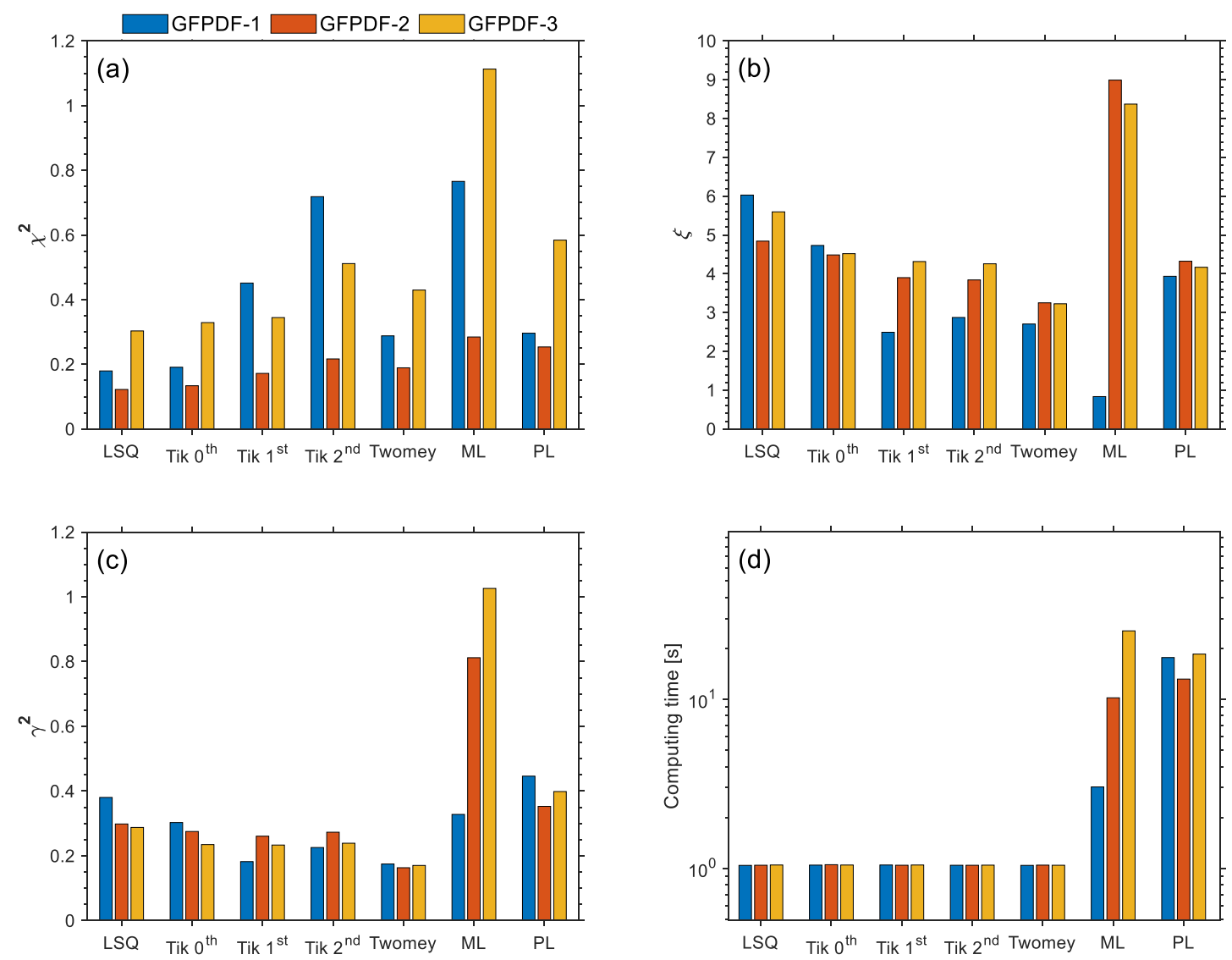

265 Figure 2. Comparison of reconstruction error, $\chi^{2}(\mathbf{a})$, the degree of smoothing, $\xi(\mathbf{b})$, the GFPDF residual, $\gamma^{2}(\mathbf{c})$ and the computing time (d) of inverted GF-PDFs using different inversion methods. Colors correspond to the pre-defined GF-PDFs with one mode (blue), two modes (orange), and three modes (yellow).

\subsection{Inversion by Twomey's method}

As Twomey's method is shown to be the best among all inversion methods examined, we examine the accuracy of inverted

GF-PDFs and the recovered mode parameters. Figure 3 compares the GF-PDFs inverted with the optimized GF and $D_{\mathrm{p}}^{*}$ bin numbers and with the pre-defined GF-PDFs. The reconstructed and the simulated HFIMS measurements are also presented in the top panel. Both the inverted GF-PDF and reconstructed HFIMS measurements are averaged over the inversions of 500 sets of synthetic data. The results demonstrate excellent agreement of the reconstructed HFIMS measurements with the synthetic data (i.e., simulated HFIMS measurements) for all three pre-defined GF-PDFs. Both the spectral shapes and peak locations of the inverted GF-PDFs agreed well with that of the pre-defined GF-PDFs. In addition, the inverted GF-PDFs are 
also in better agreement as compared with those inverted from parametric least-squares approaches (i.e., ML and PL GFPDFs, Wang et al., 2019).
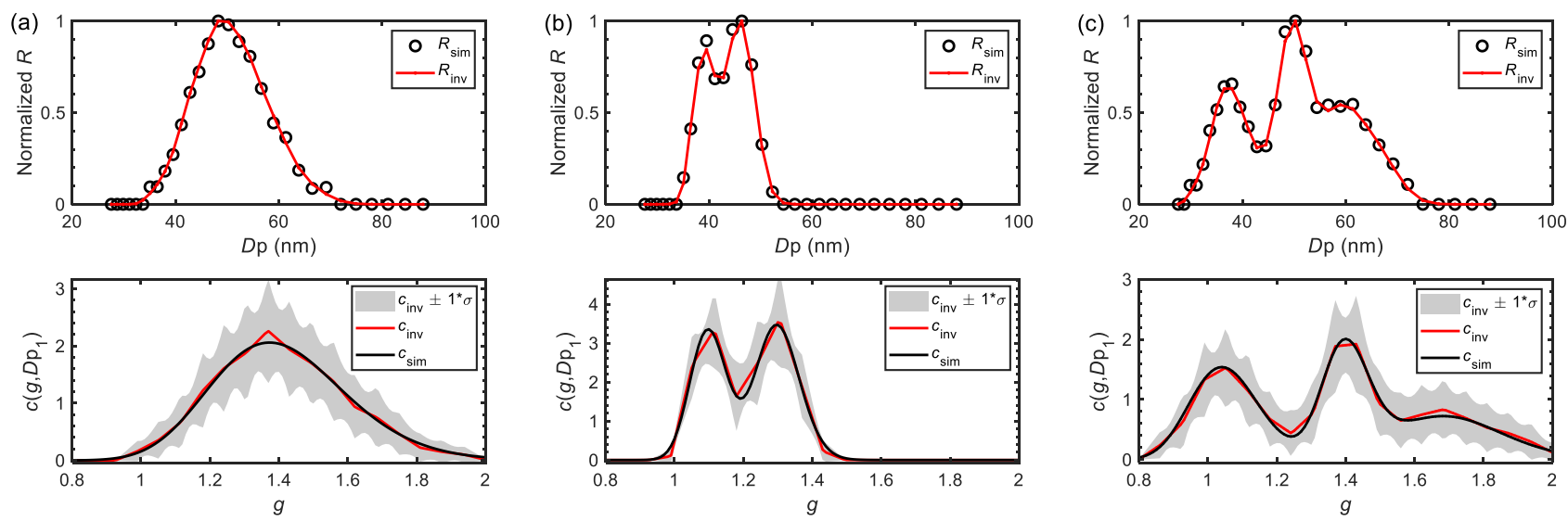

Figure 3. (Top panels) Comparisons between the averaged reconstructed HFIMS measurements and the simulated HFIMS measurements corrupted with Poisson noises for pre-defined GF-PDFs of one mode (a), two modes (b), and three modes (c), respectively. (Bottom panels) Comparisons between the pre-defined GF-PDFs and the GF-PDFs inverted using Twomey's method with the optimized value of GF bins. The shaded area represents GF-PDF solution spaces within one standard deviation.

To quantify the accuracy of the inverted GF-PDFs, we fitted the inverted GF-PDFs to recover the mode parameters as shown in Table 1. The pre-set mode parameters of the pre-defined GF-PDFs are shown in Table 1. The results show that both the mode geometric means and the multimodal number fractions can be recovered accurately with minor uncertainties.

Table 2. Recovered mode parameters of pre-defined GF-PDFs from inverted GF-PDFs.

\begin{tabular}{cccccccccc}
\hline $\begin{array}{c}\text { Predefined } \\
\text { GF-PDF }\end{array}$ & \multicolumn{3}{c}{ Mode 1 } & \multicolumn{1}{c}{ Mode 2 } & \multicolumn{3}{c}{ Mode 3 } \\
\hline 1 & $1.00 \pm 0$ & $1.39 \pm 0.03$ & $1.09 \pm 0.01$ & $f$ & $G$ & $\sigma$ & $f$ & $G$ & $\sigma$ \\
2 & $0.46 \pm 0.10$ & $1.10 \pm 0.02$ & $1.02 \pm 0.01$ & $0.54 \pm 0.10$ & $1.30 \pm 0.02$ & $1.03 \pm 0.01$ & & NA & NA \\
3 & $0.37 \pm 0.09$ & $1.05 \pm 0.03$ & $1.05 \pm 0.02$ & $0.34 \pm 0.13$ & $1.40 \pm 0.03$ & $1.03 \pm 0.02$ & $0.28 \pm 0.12$ & $1.69 \pm 0.08$ & $1.06 \pm 0.03$ \\
\hline
\end{tabular}

\section{Conclusion}

In this study, we develop and evaluate nonparametric regularized methods for inverting GF-PDF from HFIMS measurements. The integrated response of HFIMS, which is a convolution of the aerosol hygroscopic GF-PDF, the transfer function of the DMA, and the transfer function of the WFIMS, is first cast into a matrix form. With the matrix form, nonparametric regularized methods can be applied straightforwardly to invert the GF-PDF. Synthetic HFIMS measurements are generated using Monte-Carlo simulations for representative aerosol GF-PDFs, and the synthetic data are used to investigate the dependence of inverted GF-PDF on the number of GF bins (i.e., GF resolutions) and the performances of different inversion methods. We show an optimal GF bin number of 20 for all nonparametric methods and representative 
295 GF-PDFs. The performances of unregularized least-squares, Twomey's algorithm, Tikhonov regularizations, and commonly used parametric inversion methods (i.e., ML and PL least-squares fitting) are compared. Nonparametric methods based on the matrix form have substantial advantages in the inversion of GF-PDF over the parametric fitting methods as (1) no prior assumption of GF-PDF distributions is required; (2) the matrix-based form facilitates the application of different regularizations (e.g., Tikhonov regularization and Twomey's iterative regularization), which reduce the error in inverted GF-

300 PDF by eliminating noise amplification; (3) they are much more computationally efficient once the matrix is generated. On average, The GF-PDF inverted using Twomey's method has the smallest error compared to solutions using the other inversion methods regardless of the shapes of the pre-defined GF-PDFs, and it accurately reproduces the true GF-PDF, including the mode parameters and other key statistics.

305 Data availability. Datasets and code packages related to this paper will be provided by the corresponding author (Jian Wang, jian@wustl.edu) upon request.

Author contributions. JZ and JW designed the study. JZ developed the code. JZ and JW prepared the manuscript with contributions from all co-authors.

Competing interests. The authors declare that they have no conflict of interest.

310 Acknowledgements. We acknowledge the funding support from the U. S. Department of Energy's Small Business Innovation Research (SBIR) program under contract DE-SC0013103 and Small Business Technology Transfer (STTR) program under contract DESC0006312.

\section{References}

315 Collins, D. R., Flagan, R. C., and Seinfeld, J. H.: Improved Inversion of Scanning DMA Data, Aerosol Science and Technology, 36, 1-9, 10.1080/027868202753339032, 2002.

Gysel, M., Crosier, J., Topping, D. O., Whitehead, J. D., Bower, K. N., Cubison, M. J., Williams, P. I., Flynn, M. J., McFiggans, G. B., and Coe, H.: Closure study between chemical composition and hygroscopic growth of aerosol particles during TORCH2, Atmos. Chem. Phys., 7, 6131-6144, 10.5194/acp-7-6131-2007, 2007.

Gysel, M., McFiggans, G. B., and Coe, H.: Inversion of tandem differential mobility analyser (TDMA) measurements, Journal of Aerosol Science, 40, 134-151, https://doi.org/10.1016/j.jaerosci.2008.07.013, 2009.

325 Hansen, P. C.: Analysis of Discrete Ill-Posed Problems by Means of the L-Curve, SIAM Review, 34, 561-580, 10.1137/1034115, 1992.

Hansen, P. C., and O'Leary, D. P.: The Use of the L-Curve in the Regularization of Discrete Ill-Posed Problems, SIAM Journal on Scientific Computing, 14, 1487-1503, 10.1137/0914086, 1993. 
Hansen, P. C.: REGULARIZATION TOOLS: A Matlab package for analysis and solution of discrete ill-posed problems, Numerical Algorithms, 6, 1-35, 10.1007/BF02149761, 1994.

Kandlikar, M., and Ramachandran, G.: INVERSE METHODS FOR ANALYSING AEROSOL SPECTROMETER 8502(98)00066-4, 1999.

Liu, B. Y. H., Pui, D. Y. H., Whitby, K. T., Kittelson, D. B., Kousaka, Y., and McKenzie, R. L.: The aerosol mobility chromatograph: A new detector for sulfuric acid aerosols, Atmospheric Environment (1967), 12, 99-104, https://doi.org/10.1016/0004-6981(78)90192-0, 1978.

Markowski, G. R.: Improving Twomey's Algorithm for Inversion of Aerosol Measurement Data, Aerosol Science and Technology, 7, 127-141, 10.1080/02786828708959153, 1987.

345 Olfert, J. S., Kulkarni, P., and Wang, J.: Measuring aerosol size distributions with the fast integrated mobility spectrometer, Journal of Aerosol Science, 39, 940-956, https://doi.org/10.1016/j.jaerosci.2008.06.005, 2008.

Petters, M. D.: A Software Package to Simplify Tikhonov Regularization with Examples for Matrix-Based Inversion of SMPS and HTDMA Data, Atmos. Meas. Tech. Discuss., 2021, 1-27, 10.5194/amt-2021-51, 2021.

Pilinis, C., Pandis, S. N., and Seinfeld, J. H.: Sensitivity of direct climate forcing by atmospheric aerosols to aerosol size and composition, Journal of Geophysical Research: Atmospheres, 100, 18739-18754, https://doi.org/10.1029/95JD02119, 1995.

Pinterich, T., Spielman, S. R., Hering, S., and Wang, J.: A water-based fast integrated mobility spectrometer (WFIMS) with enhanced dynamic size range, Aerosol Science and Technology, 51, 1212-1222, 10.1080/02786826.2017.1338664, 2017a.

Pinterich, T., Spielman, S. R., Wang, Y., Hering, S. V., and Wang, J.: A humidity-controlled fast integrated mobility spectrometer (HFIMS) for rapid measurements of particle hygroscopic growth, Atmos. Meas. Tech., 10, 4915-4925, 10.5194/amt-10-4915-2017, 2017b.

Rader, D. J., and McMurry, P. H.: Application of the tandem differential mobility analyzer to studies of droplet growth or evaporation, Journal of Aerosol Science, 17, 771-787, https://doi.org/10.1016/0021-8502(86)90031-5, 1986.

Rawat, V. K., Buckley, D. T., Kimoto, S., Lee, M.-H., Fukushima, N., and Hogan, C. J.: Two dimensional size-mass 365 distribution function inversion from differential mobility analyzer-aerosol particle mass analyzer (DMA-APM) measurements, Journal of Aerosol Science, 92, 70-82, https://doi.org/10.1016/j.jaerosci.2015.11.001, 2016.

Sipkens, T. A., Olfert, J. S., and Rogak, S. N.: Inversion methods to determine two-dimensional aerosol mass-mobility distributions: A critical comparison of established methods, Journal of Aerosol Science, 140, 105484, 370 https://doi.org/10.1016/j.jaerosci.2019.105484, 2020.

Stolzenburg, M., and McMurry, P.: TDMAFIT user's manual, University of Minnesota, Department of Mechanical Engineering, Particle Technology Laboratory, Minneapolis, 1-61, 1988.

375 Stolzenburg, M. R., and McMurry, P. H.: Equations Governing Single and Tandem DMA Configurations and a New Lognormal Approximation to the Transfer Function, Aerosol Science and Technology, 42, 421-432, 10.1080/02786820802157823, 2008. 
Swietlicki, E., Hansson, H.-C., Hämeri, K., Svenningsson, B., Massling, A., McFiggans, G., McMurry, P. H., Petäjä, T., 380 Tunved, P., and Gysel, M.: Hygroscopic properties of submicrometer atmospheric aerosol particles measured with H-TDMA instruments in various environments - a review, Tellus B: Chemical and Physical Meteorology, 60, 432-469, 2008.

Talukdar, S. S., and Swihart, M. T.: An Improved Data Inversion Program for Obtaining Aerosol Size Distributions from Scanning Differential Mobility Analyzer Data, Aerosol Science and Technology, 37, 145-161, 10.1080/02786820300952, 3852003

Tang, I., and Munkelwitz, H.: Aerosol phase transformation and growth in the atmosphere, Journal of Applied Meteorology, 33, 791-796, 1994.

390 Twomey, S.: Comparison of constrained linear inversion and an iterative nonlinear algorithm applied to the indirect estimation of particle size distributions, Journal of Computational Physics, 18, 188-200, https://doi.org/10.1016/00219991(75)90028-5, 1975.

Wang, Y., Pinterich, T., and Wang, J.: Rapid measurement of sub-micrometer aerosol size distribution using a fast integrated mobility spectrometer, Journal of Aerosol Science, 121, 12-20, https://doi.org/10.1016/j.jaerosci.2018.03.006, 2018.

Wang, Y., Zheng, G., Spielman, S. R., Pinterich, T., Hering, S. V., and Wang, J.: Retrieval of high time resolution growth factor probability density function from a humidity-controlled fast integrated mobility spectrometer, Aerosol Science and Technology, 53, 1092-1106, 2019.

Zhang, J., Spielman, S., Wang, Y., Zheng, G., Gong, X., Hering, S., and Wang, J.: Rapid measurement of RH-dependent aerosol hygroscopic growth using a humidity-controlled fast integrated mobility spectrometer (HFIMS), Atmos. Meas. Tech., 14, 5625-5635, 10.5194/amt-14-5625-2021, 2021.

405 Zheng, G., Kuang, C., Uin, J., Watson, T., and Wang, J.: Large contribution of organics to condensational growth and formation of cloud condensation nuclei $(\mathrm{CCN})$ in the remote marine boundary layer, Atmospheric Chemistry and Physics, 20, 12515-12525, 2020. 